\title{
Determinación de la presencia de Salmonella spp; Staphylococcus aureus; Campylobacter jejuni y Campylobacter coli en productos cárnicos procesados en Cabañas y Cuscatlán
}

\author{
Ana Elizabeth Perlera de Escalante ${ }^{1}$
}

Shigeo Nishino ${ }^{2}$

1. Licenciada en Química y Farmacia con especialidad en Microbiología, docente Facultad de Ingeniería y Arquitectura, Facultad Multidisciplinaria de Ilobasco, Universidad Católica de El Salvador, ana.perlera@catolica. edu.sv

2. Médico veterinario con Especialidad en Sanidad Animal e Inspección de carne, voluntario Senior de JICA, shige_kota_reiko@yahoo.co.jp

Recepción: 2014-04-20

Aceptación: 2014-05-22

\section{Resumen}

La investigación presenta un análisis sobre la determinación de la presencia de Salmonella spp; Staphylococcus aureus; Campylobacter jejuni y Campylobacter coli en los productos cárnicos de mayor consumo entre la población de los departamentos de Cuscatlán y Cabañas, pertenecientes a la Zona Central de El Salvador. El estudio se desarrolló mediante el muestreo de productos cárnicos cocidos y crudos, así como a través de la utilización de carne de pollo como materia prima. Sin embargo, este recurso corre el riesgo de sufrir contaminación cruzada, la cual puede efectuarse en los tiempos de preparación del alimento, por medio de Campylobacter. Esta bacteria se traslada desde los intestinos del ave a la piel del mismo y, por consiguiente, a los procesos de manufactura de los productos cárnicos.

Se demostró la presencia de Salmonella spp; Staphylococcus aureus; Campylobacter jejuni y Campylobacter coli, en ambos departamentos de estudio, cuya procedencia correspondió a lugares en donde la carne cruda de pollo no es llevada bajo cadenas de frío; ni los procesos de empacado y almacenamiento son adecuados para cárnicos crudos y/o cocidos. Asimismo, se sientan precedentes de identificación por contaminación microbiológica para este tipo de alimentos.

Palabras clave: Cadena de frío, Salmonella spp; Staphylococcus aureus; Campylobacter jejuni y Campylobacter coli, cárnicos crudos y cocidosAbstract

\begin{abstract}
This research presents the analysis of determining the presence of Salmonella spp; Staphylococcus aureus; Campylobacter jejuni y Campylobacter coli in meat products of high consumption among people living in Cuscatlán and Cabañas that are located in the central area of El Salvador. The study was developed through a sample of cooked and raw meat products; as well as through using chicken meat as raw material. However, this resource takes the risk of having crosscontamination that can be carried out when preparing the food through Campylobacter. This bacteria was taken from the bird's intestines to its skin and, as a consequence, to the processes of manufacturing of the meat products

It was demonstrated the presence of spp; Staphylococcus aureus; Campylobacter jejuni y Campylobacter coli, in both towns whose origin matched the places where the raw meat of the chicken is not taken from low chains of coldness; nor the packing and storing processes that are adequate of the cooked and/or meat. At the same time, this research presents an antecedent to identify the microbiologic pollution for these kinds of foods.
\end{abstract}

Key words: Cold chain, Salmonella spp; Staphylococcus aureus; Campylobacter jejuni y Campylobacter coli, cooked and raw meat 


\section{Introducción}

La contaminación en la producción y comercialización de alimentos derivados de carnes de cerdo, pollo, y res son algunas de los principales causas de las infecciones de tipo alimentarias que producen gastroenteritis. Por ejemplo: salmonelosis (originada por Salmonella spp) realizando pruebas de identificación para cada análisis presuntivo, por existir varios tipos como lo menciona para Crossa, Brenner y Ewing (1973) ha expresado en el estudio de la relación secuencial de polinucleótidos para determinar la diferencia entre las diferentes cepas de Salmonella en relación con otras bacterias entéricas, su evolución y diferencias entre sí; para ello Le Minor, Michel y Popoff (1987) describen la nomenclatura para los tipos de Salmonella y su taxonomía según el papel clínico de las cepas. También la campilobacteriosis es una infección originada por Campylobacter jejuni y Campylobacter coli, según Berndtson, Tivemo y Engvall (1992), mencionan que esta bacteria presente en el tracto intestinal se puede transmitir a las canales de pollo durante el proceso de sacrificio, por ello es propicio a la contaminación por los manipuladores y finalmente la intoxicación por Staphylococcus aureus. Estas enfermedades generan problemas de salud a los consumidores, así como también gastos en las economías familiares y en el presupuesto del Estado por lo que se invierte en sus respectivos tratamientos.
Esta investigación tuvo como objetivo determinar la presencia de organismos patógenos en productos cárnicos procesados y/o comercializados en los departamentos de Cabañas y Cuscatlán, pertenecientes a la Zona Central de El Salvador. Esto permitió la recolección de datos y generación de información necesaria sobre la calidad e inocuidad de alimentos, para que las autoridades competentes determinen las medidas paliativasy de monitoreo pertinentes, en el procesamiento y comercialización de dichos productos.

Como parte de la investigación fue necesaria la recolección de muestras de diferentes centros de procesamiento y comercialización, las cuales fueron sometidas a diferentes técnicas y tratamientos de laboratorio, según detalla para Berdoll, M. (1984) en los métodos de análisis correspondiente a la siembra de muestras de alimentos en los medios de cultivo selectivos, a fin de determinar la presencia de los organismos patógenos en cuestión.

La investigación se llevó a cabo durante tres etapas: Ensayo de la investigación en cuanto al comportamiento de las pruebas preliminares; análisis de procesos y productos; y finalmente, seguimiento del mismo de acuerdo al índice de presencia de los organismos patógenos. 


\section{Materiales y métodos}

El estudio se abordó de forma experimental, para Bennet, R. (1984) se deben acordar los procesos al FDA, que establece el tipo y cantidad de muestra necesaria para laboratorio, de acuerdo a su origen $y$ procedimiento de elaboración, ya que se determinó la presencia de Salmonella spp; Staphylococcus aureus; Campylobacter jejuni y Campylobacter coli en cárnicos crudos y cocidos; en relación al caso mencionamos que «fue necesario aislar alrededor de 1000 muestras para la identificación de los diferentes tipos de cepas de Campylobacter, incluyendo muestras en humanos, pollo, oveja, cerdo, ganado, vísceras y agua durante 18 meses» (Bolton, 1984). El análisis se llevó acabo en el laboratorio de microbiología de los alimentos de la Facultad Multidisciplinaria de Ilobasco, utilizando materiales y medios de cultivo correspondientes a los procedimientos establecidos para su preparación como lo menciona Aspinal, Wareing, Hayward y Hutchinson en la implementación de sangre en la preparación de medios de cultivo. Dichos procesos realizados en la sede regional de la Universidad Católica de El Salvador, ubicada en el municipio de Ilobasco, Cabañas.

El proceso de análisis constó de tres etapas de identificación: las primeras dos se realizaron solo para cárnicos crudos y cocidos, en tres establecimientos de la zona de Cuscatlán; y dos establecimientos en Cabañas. Finalmente, la tercera etapa para análisis de Campylobacter jejuni y Campylobacter coli se utilizó carne de pollo como materia prima, para Jacob-Reitsma, Lyhs y Wagenaar (2000) establece análisis para la carne de pollo, donde representa un riesgo potencial por los diferentes tipos de contaminación que sufre, a partir de los procesos de obtención de la carne, y de la manipulación para los procesos de productos de pollo. Las técnicas para el desarrollo de los análisis de muestras cárnicas se llevaron a cabo de acuerdo a las metodologías del laboratorio utilizado; en relación a la investigación es importante tomar en cuenta como lo considera Omoe $y$ otros (2003) la toxicidad que presentan los microorganismos en estudio, para el que menciona el Staphilococcus aureus y su forma de crecimiento aún en otros medios de cultivo. Es importante apoyarse para cada una de las etapas en la referencias que nos ofrecen los autores, tanto en métodos y técnicas según Doyle P. (2001), como en la morfología de crecimiento para tomar en cuenta las características obtenidas, como se refiere para Varnam A. (s.f.)

\section{Resultados y discusión}

Las técnicas aplicadas para el desarrollo de la investigación consistieron en metodologías de análisis microbiológico para alimentos. Para el caso, al dividir el estudio en tres etapas 
de ejecución - de acuerdo a la cantidad de muestras procesadas - se observó fácilmente que los tipos de productos fueron elegidos en proporciones diferentes, según los volúmenes de producción de la microempresa cárnica.

\section{a. Etapa I}

Para la realización del estudio se utilizaron cinco diferentes tipos de cárnicos, especialmente aquellos que figuraban entre la preferencia de la población el departamento de Cabañas.
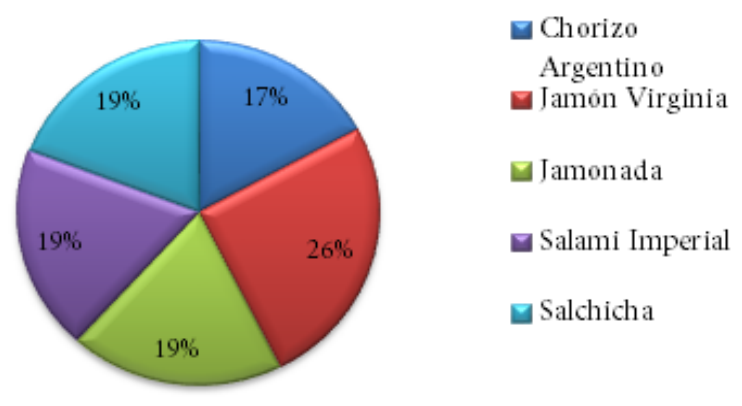

Figura 1. Gráfico con porcentajes de muestras analizadas en la etapa I, Cabañas.

El gráfico muestra cada una de las especialidades cárnicas seleccionadas para la identificación de cada uno de los microorganismos, correspondientes al estudio, y cuya presencia se deseaba comprobar.

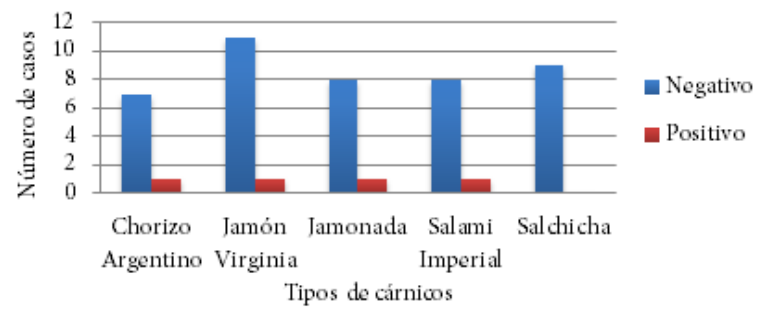

Figura 2. Gráfico que muestra la presencia de Salmonella spp en cárnicos. Etapa I, Cabañas.

En el gráfico se muestran el número de casos de Salmonella spp, analizados, donde se observar la existencia de casos positivos respectivamente. Sin embargo, se registraron más casos de ausencia que de presencia del microorganismo; siendo el jamón Virginia la muestra con más casos negativos (11 casos), en comparación al chorizo argentino (7). Estas cifras suponen la existencia de una adecuada vigilancia dentro de los procesos de manufactura.

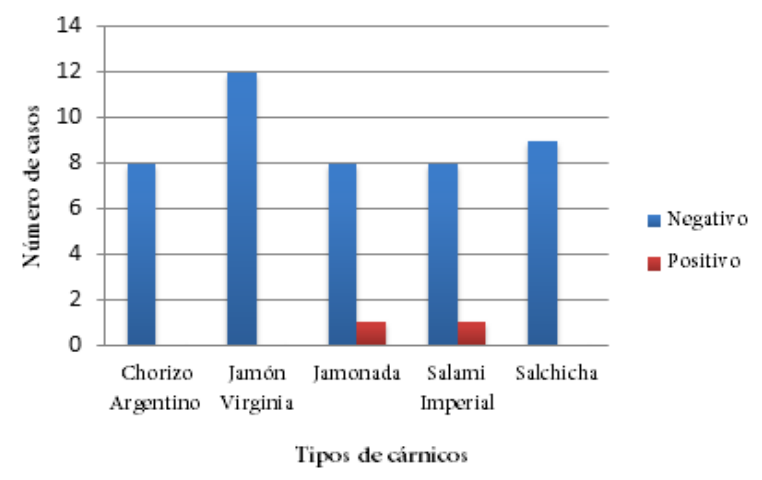

Figura 3. Gráfico de pruebas de Staphylococcus aureus en I Etapa, Cabañas.

Para el gráfico 3, correspondiente a Staphylococcus aureus, muestran los casos negativos, para el presente el jamón Virginia despunta en cuanto a mayor cantidad de resultados negativos (12 casos), a diferencia 
de cuatro puntos a los productos de chorizo argentino, jamonada y salami imperial. Sin embargo, estos dos últimos mostraron una presencia positiva leve en cuanto a esta bacteria. Esto puede considerarse normal, ya que la normativa salvadoreña obligatoria (NSO) de Carne y productos Cárnicos, Embutidos Crudos y Cocidos NSO RTCA 67.01.33:06, estable rangos de presencia de Staphylococcus aureus en los productos, los cuales limitan la ingesta de los mismos para el bienestar de los consumidores.

\section{b. Etapa II}

En el desarrollo de la II etapa, que correspondió a productos cárnicos crudos y cocidos de los departamentos de Cabañas y Cuscatlán, se tomó una muestra de seis tipos de cárnicos.
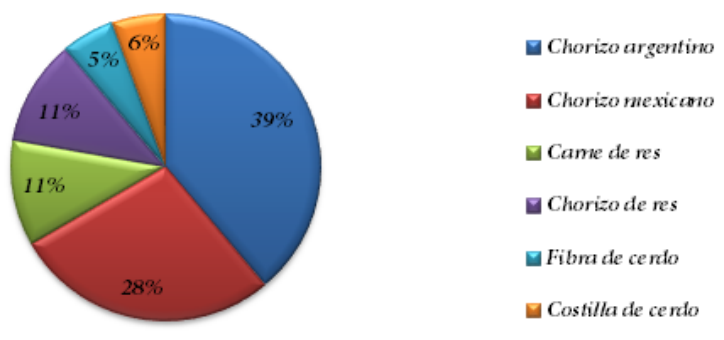

Figura 4. Gráfico que muestra los productos analizados en Cabañas, II Etapa.

En el gráfico la mayor cantidad de la muestras corresponden a chorizo argentino (39\%), seguido del chorizo mexicano (28\%). Los productos analizados en menor cantidad fueron la fibra (5\%) y costilla de cerdo (6\%), respectivamente, cada especialidad refleja las especialidades de productos cárnicos preferentemente consumidos.

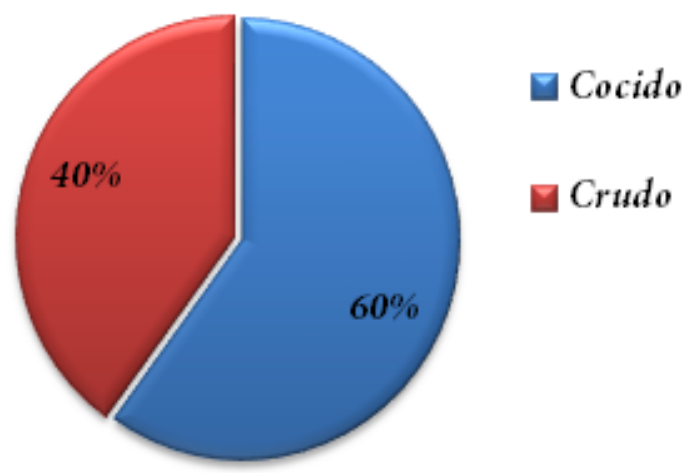

Figura 5. Gráfico de clasificación de productos analizados en Cabañas, II Etapa.

Se puedeobservar con facilidad los porcentajes de muestras analizadas, según el tipo de producto cárnico, ya sea crudo o cocido, el último con el porcentaje más alto; según se toma en cuenta que las pruebas realizadas a las muestras tomadas en el departamento de Cabañas, durante la segunda etapa, solo el 5\% de ellas resultó positivo para Salmonella spp. Esto indicó una posible y leve contaminación cruzada en el proceso de manufactura de los productos. Sin embargo, la presencia de Staphylococcus en la misma muestra fue nula, lo que hace pensar que existió un manejo adecuado del empacado y distribución del producto terminado.

Para la muestra utilizada dentro de la Etapa II, en el departamento de Cuscatlán, se seleccionaron diversos productos cárnicos, específicamente aquellos de mayor consumo y popularidad en ese lugar. Se determinó que los cárnicos preferidos por la población 
tienden a ser crudos (52\%), debido a que poseen una amplia variedad. No obstante, los productos cocidos (26\%) y ahumados (22\%), también tienen presencia entre los habitantes de Cuscatlán.
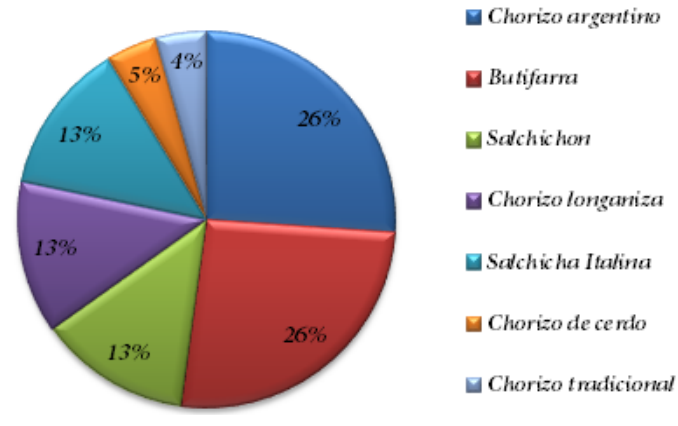

Figura 6. Gráfico de productos analizados en el departamento de Cuscatlán, etapa II.

Para el presente gráfico, se procedió a realizar la determinación de la presencia de las bacterias, la Salmonella spp quien refleja el porcentaje más bajo (5\%), demostrando que las prácticas de manufactura siguen siendo poco adecuadas. De acuerdo a lo establecido por la normativa salvadoreña para productos cárnicos (NSO RTCA 67.01.33:06). Por su parte, el Staphilococcus aureus se encontró en mayor cantidad dentro de las muestras (9\%).

\section{c. Etapa III}

A diferencia de las dos primeras etapas, para esta última fase se utilizó pollo crudo como muestra de estudio, a fin de determinar la presencia de Campylobacter. Específicamente esta materia prima requiere una observancia minuciosa dentro de la elaboración de productos cárnicos, por el tipo de contaminación que puede ser generada por ella misma, y a la cual se ve expuesta durante los procesos, Genigiorgis y otros (1986) mencionan que una de las causas que contribuyen a la contaminación corresponde a la falta de saneamiento efectiva al final de la jornada en las plantas de sacrificio. Ante las posibles causas que han determinado la presencia de Campylobacter en 2007, Son I. menciona al respecto «los casos analizados por prevalencia de la bacteria en las canales de pollo de engorde se encuentra en un $78.5 \%$ en las aves sacrificadas, y según el análisis por PCR (Reacción en cadena por Polimerasa), que los tipos de Campylobacter fueron pertenecientes a los tipos jejuni y coli».

Luego de realizarse las pruebas pertinentes, se identificó la presencia de esta bacteria en un $60 \%$ de las muestras. En los productos avícolas, procedentes del departamento de Cuscatlán, la cifra positiva para Campylobacter fue de $58 \%$. Este porcentaje hace referencia a los procesos incorrectos que son implementados para quitarle la piel al pollo, y que pueden generar intoxicaciones alimentarias. Por su parte, para el departamento de Cabañas, la incidencia de contaminación fue superior (63\%), reflejándose la necesidad de controlar el manejo de materia prima cruda. Ello incide claramente en el control de temperatura y su relación con los instrumentos usados para los procesos de producción. 
Para el caso de Staphylococcus aureus, específicamente en el departamento de Cuscatlán, un $16 \%$ de la muestra resultó ser positivo a la bacteria. Esto se refleja, en su mayoría, en las formas de manipulación del producto, especialmente en la clasificación de cárnicos crudos; cuyos preservantes se reducen únicamente a insumos naturales. Asimismo, esta bacteria dio positivo para el departamento de Cabañas con un porcentaje del 57\%.

Este dato refleja que existe un manejo inadecuado de los insumos durante las prácticas de manufactura y almacenamiento de los productos. Es notorio que los productores de pollo crudo, pertenecientes a esta zona de comercialización, tienen menos cuidado en las prácticas higiénicas.

Dentro de las 75 muestras analizadas para esta etapa III, los valores de ausencia de Staphylococcus aureus registraron una cifra significativa (68\%). Sin embargo, los niveles positivos de presencia de esta bacteria, sugieren la toma de medidas más exigentes para la manipulación de productos.

En el departamento de Cuscatlán, las pruebas realizadas para la bacteria Salmonella spp, indicaron un $27 \%$ de contaminación. Esto hace pensar que no existen las debidas medidas de control para la manipulación del material. Por otro lado, los valores negativos, reportados para el departamento de Cabañas, siguieron siendo mayores (70\%). Ello hace deducir que existe descuido en el tratamiento por frío y en los procesos de sanitación para los utensilios con los cuales se manipula la carne, ya sea para almacenar, distribuir y/o procesar.

\section{Lugares de procedencia}

$\mathrm{Al}$ analizar la relación que existe entre las muestras de carne de pollo cruda y su lugar de procedencia, uno de los factores que más llaman la atención es el proceso de obtención de la materia prima cárnica.

En la investigación se observó el corto tiempo que permanece la carne en el anaquel, al ser vendida en el mercado; en comparación al tiempo que el pollo crudo se expone en el supermercado. En este último, la carne cruda se ve expuesta a diversos utensilios y a temperaturas elevadas y/o descendentes abruptamente. Estos cambios radicales y continuos, que ocurren variadas ocasiones al día, son un factor que eleva la presencia de Campylobacter en ese tipo de producto cárnico.

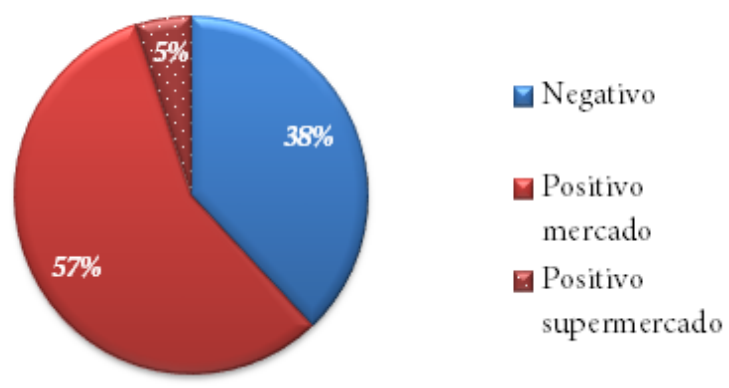

Figura 7. Pruebas de presencia de Campylobacter en Mercados y Supermercados, etapa III. 
Se observa que de las 75 muestras analizadas para la bacteria Campylobacter, tanto en el mercado como en el supermercado, el 57\% de las muestras del primero dieron positivo; mientras que solo el $5 \%$ de la carne cruda que se vende en los supermercados posee la bacteria. Solamente un 38\% de las muestras que dieron negativo.

Caso contario para la bacteria Staphylococcus, cuya presencia en el mercado fue de $21 \%$ y en los supermercados de $11 \%$; pero un $68 \%$ de las muestras resultaron negativas. Estos datos, como se pueden ver en la figura 8 , hacen pensar que existe cierta noción sobre la prevención y el tratamiento de la carne cruda de pollo en algunos lugares de comercialización.

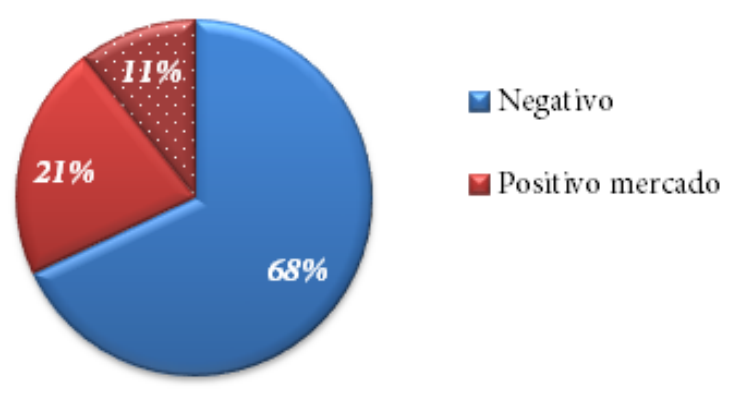

Figura 8. Pruebas de presencia de Staphylococcus en mercados y supermercados, etapa III.

En el gráfico, Salmonella spp, redujo en casos positivos, tomando en cuenta que de este microorganismo no se manifiesta para las muestras que provenían del supermercado. Esto indica que las prácticas de manipulación y prevención de contaminación o recaen simultáneamente en unafalla o son sumamente estrictas. Esto es preocupantes, pues de no existir una adecuada manipulación en la cocción, ello podría llevar a la intoxicación.

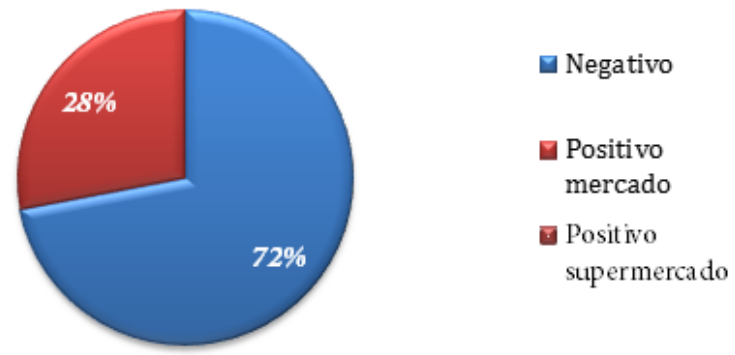

Figura 9. Pruebas de presencia de Salmonella spp en mercados y supermercados, etapa III.

Se observa claramente en el gráfico que no se registran casos positivos para las muestras provenientes del supermercado en ña identificación de Salmonella spp.

\section{Conclusiones}

Este estudio permite un primer avance en la prevención y seguridad alimentaria que debe existir dentro de todas etapas del proceso productivo; pero con mayor énfasis en el control de materia prima y los procedimientos de higiene y sanidad de los insumos utilizados. Esta información también es de utilidad para los futuros investigadores en inocuidad de alimentos, quienes pueden resaltar y/o enfocarse en los porcentajes que reflejan contaminación, a fin de determinar cuál es el origen de la misma, reconocer sus diferentes fuentes, controlarlas o prevenirlas. Asimismo, esta temática puede dar pie para iniciar una nueva investigación relacionando diferentes variables. 
En la práctica cotidiana de venta, para un microempresario, es difícil mantener un control de calidad, pues su principal objetivo es generar beneficios para su microempresa, a través de los costos invertidos. Hoy en día, la proyección de los microempresarios es formar parte del mercado formal para así competir; sin embargo, antes de iniciar el crecimiento de la empresa, se deben prever con anticipación un análisis que garanticen los procesos de manipulación, asegurando que ellos se ajustan a las medidas higiénicas de producción.

La mayoría de los problemas registrados y vinculados a los casos positivos o de presencia de los microorganismos, están estrechamente relacionados con el tipo de formación o capacitación a la que pueden accesar quienes manipulan el cárnico. De esta manera, la formación e impulso de talleres cumplen una función primordial dentro de la instrucción adecuada para procesar los cárnicos.

Como institución esto corresponde una labor continua más comprometida para facilitar a los productores una correcta formación, en cuanto al adecuado manejo y ejecución de los planes de limpieza y desinfección de la carne de cualquier tipo. También se pueden orientar planes hacia la resolución de problemas que originan la contaminación del producto terminado. Para ello, los profesionales técnicos de la zona deben convertirse en agentes de cambio. Es decir brindar asistencia auxiliar o de tiempo completo para cada uno de los productores que soliciten de su ayuda.

Este objetivo cumple con la visión institucional de formar profesionales capaces de resolver problemassociales, yde maximizar el potencial que cada uno de los microempresarios emprenden constantemente. Sin embargo, el desconocimiento es una de las razones por las cuales actualmente no es solicitada una ayuda técnica para la mejora de los procesos. A través de prácticas sencillas que no requieren de capitales elevados de inversión, se pueden producir cambios significativos.

Por otra parte, los departamentos de Cuscatlán y Cabañas poseen altos índice de actividad productiva en cuanto a productos cárnicos, específicamente en el área no formal. No obstante, no existe información suficiente que ayude a guiar a los pequeños productores para mejorar los procesos y manejo de los mismos. Esta realidad hace necesaria la existencia de un registro que guíe el mejoramiento del proceso de producción de los productos consumidos.

Es importante considerar la prevención y las buenas prácticas higiénicas dentro de cada uno de los procesos de elaboración de productos cárnicos, a fin de generar concientización y mejoría en los mismos. 
No obstante, dentro del Sistema Nacional de Salud de El Salvador, cuando se registran casos de enfermedades gastrointestinales, no se especifica el origen de las mismas. De esta forma no existen precedentes que validen o nieguen la incidencia de las bacterias Staphylococcus aureus, Salmonella spps o Campylobacter coli, jejuni como causas de estos padecimientos.
En la investigación se supuso- de forma previa - la existencia de contaminación por causa del ambiente dentro de cada una de las muestras. Por ello contempló la presencia de aerobios mesófilos y coliformes E. coli, los cuales fueron encontrados dentro del análisis para Campylobacter:

\begin{tabular}{|c|c|c|c|}
\hline Nombre & Cantidad $^{3}$ & $\begin{array}{l}\text { Número de } \\
\text { casos }\end{array}$ & $\begin{array}{c}\text { Lugar de } \\
\text { procedencia }\end{array}$ \\
\hline \multirow{5}{*}{$\begin{array}{l}\text { Aerobios } \\
\text { mesófilos }\end{array}$} & De $1,000-5,00 \mathrm{UFC} / \mathrm{g}$ & 2 & Mercado \\
\hline & De $50,000-100,000 \mathrm{UFC} / \mathrm{g}$ & 2 & Mercado \\
\hline & De $150,000-990,000 \mathrm{UFC} / \mathrm{g}$ & 16 & $\begin{array}{c}\text { Mercado (7) } \\
\text { Supermercado (9) }\end{array}$ \\
\hline & $\begin{array}{c}\text { De } 1,000,000-2,000,000 \\
\text { UFC/g }\end{array}$ & 10 & Mercado \\
\hline & Mayor a 2,000,000 UFC/g & 42 & $\begin{array}{c}\text { Mercado (39) } \\
\text { Supermercado (3) }\end{array}$ \\
\hline
\end{tabular}

Figura 10. Presencia de patógenos dentro de las muestras, de acuerdo al lugar de la recolección.

\begin{tabular}{|c|c|c|c|}
\hline Nombre & \multicolumn{1}{|c|}{ Cantidad $^{5}$} & Número de casos & Lugar de procedencia $^{6}$ \\
\hline & $0-100 \mathrm{UFC/g}$ & 56 & $\begin{array}{c}\text { Mercado (44) } \\
\text { Supermercado (12) }\end{array}$ \\
\cline { 2 - 4 } Coliformes & $100-200 \mathrm{UFC/g}$ & 3 & $\begin{array}{c}\text { Mercado (2) } \\
\text { Supermercado (1) }\end{array}$ \\
\cline { 2 - 4 } & $200-300 \mathrm{UFC/g}$ & 9 & $\begin{array}{c}\text { Mercado (7) } \\
\text { Supermercado (2) }\end{array}$ \\
\cline { 2 - 4 } & Mayor a 300 UFC/g & 7 & Mercado \\
\hline
\end{tabular}

Figura 11. Presencia de patógenos dentro de las muestras, de acuerdo al lugar de la recolección. 
Los datos expuestos reflejan el grado de contaminación ambiental que existe en cada uno de los lugares estudiados dentro de la investigación. Es evidente que, en algunos lugares más que en otros, se requiere de la adecuada y metódica implementación de procesos integrados de higiene y sanitación, para evitar que la presencia de los agentes afecte el producto; y por ende, el consumo humano.

Conocer la cantidad y los tipos de agentes microbiológicos que se encuentran en la zona de investigación, conduce a los futuros profesionales en el área de alimentos, hacia una responsabilidad social más fuerte.

Las bacterias entéricas son de origen intestinal. En el caso de la carne de pollo que sirvió como muestra para el estudio, se percibe una posible contaminación por inadecuada manipulación del producto. Esto sería lo que provocó encontrar Campylobacter y Salmonella spp, en ellos. Los niveles encontrados pueden incrementarse, debido a que estas bacterias son de la misma naturaleza que los coliformes.

Para las próximas investigaciones, estos aspectos servirán como insumos en la búsqueda de soluciones a los problemas de inocuidad y del control de calidad, que exige para cada producto cárnico crudo o cocido; y también cualquier otro tipo de alimento derivado de la carne de res, de cerdo o de pollo.

\section{Referencias}

Aspinall, S; y otros (1993). Selective medium for Thermophilic Campylobacters including Campylobacter upsaliens.

Bennet, R; (1984). Bacteriological Analytical Manual, Food and Drugs Administration (FDA). Association of Official Analytical Chemists. 6th Edition.

Berdoll, M; y otros (1984). Compendius of methods for the microbiological examination of methods for the microbiological examination of foods. American Public Health Association Washignton, D.C., 2nd edition.

Berndtson, E; y otros (1992). Distribution and numbers of Campylobacter in newly slaughtered broiler chickens and hens. Food Mocribiology.

Bolton, F; y otros (1984). Campylobacter biotyping scheme of epidemiological value. Research Article 34, 956. 115. 307 
Crossa, J; y otros. (1973). Molecular relationship among the salmonella. J. Bacteriol 307-315.

Doyle P. (2001). Microbiología de los Alimentos, bacterias patógenas. Editorial Acribia . págs. 139,167 y 382 ,

Genigeorgis, C; y otros (1986). Campylobacter jejuni infection on poultry farms and its effect on poultry meat contamination during slaughtering. 895-903

Le Minor, L; y otros (1987). Designation of Salmonella enteric sp. nov. nom. rev. as the type and only species of the genus Salmonella.

Newell, D; y otros (2000). Poultry infection and their control at the farm level, Campylobacter. 2nd edition, 497-509, ASM press Washington DC.

Omoe, K; y otros (2003). Characterization of Novel Staphylococcal Enterotoxine-Like toxin type "P". Infect Immune 711, 6088.

Reitsma, W; y otros (2000). Campylobacter in the food supply, 2nd edition, 627-644 ASM press Washington DC.

Son I; y otros (2007). Prevalence of Arcobacter and Campylobacter on broiler carcasses during processing. Food Microbiology 113, 16-22

Varnam, A; y otros. (s.f.). Foodborne pathogens, an illustrated text, Manson publishing, UK. ISBN: 1874545413. 